\title{
Cover Contact Graphs
}

Nieves Atienza ${ }^{2, \star}$, Natalia de Castro ${ }^{2, \star}$, Carmen Cortés $^{2, \star}$, M. Ángeles Garrido ${ }^{2, \star}$, Clara I. Grima ${ }^{2, \star}$, Gregorio Hernández ${ }^{1}$, Alberto Márquez ${ }^{2, \star}$, Auxiliadora Moreno ${ }^{2, \star}$, Martin Nöllenburg $^{3, \star \star}$, José Ramon Portillo ${ }^{2, \star}$, Pedro Reyes ${ }^{2, \star}$, Jesús Valenzuela ${ }^{2, \star}$, Maria Trinidad Villar ${ }^{2, \star}$, and Alexander Wolff ${ }^{4}$

${ }^{1}$ Dept. Matemática Aplicada, Fac. Informática, Univ. Politécnica de Madrid, Spain gregorio@fi.upm.es

${ }^{2}$ Universidad de Sevilla, Spain

\{natienza, natalia, ccortes, vizuete, grima, almar, auxiliadora, josera, preyes, jesusv, villar\}@us.es

${ }^{3}$ Fakultät für Informatik, Universität Karlsruhe, Germany noelle@ira.uka.de

${ }^{4}$ Faculteit Wiskunde en Informatica, TU Eindhoven, The Netherlands http: //www.win.tue.nl/ awolff

\begin{abstract}
We study problems that arise in the context of covering certain geometric objects (so-called seeds, e.g., points or disks) by a set of other geometric objects (a so-called cover, e.g., a set of disks or homothetic triangles). We insist that the interiors of the seeds and the cover elements are pairwise disjoint, but they can touch. We call the contact graph of a cover a cover contact graph (CCG). We are interested in two types of tasks: (a) deciding whether a given seed set has a connected $\mathrm{CCG}$, and (b) deciding whether a given graph has a realization as a CCG on a given seed set. Concerning task (a) we give efficient algorithms for the case that seeds are points and covers are disks or triangles. We show that the problem becomes NP-hard if seeds and covers are disks. Concerning task (b) we show that it is even NP-hard for point seeds and disk covers (given a fixed correspondence between vertices and seeds).
\end{abstract}

\section{Introduction}

Koebe's theorem 911, a beautiful and classical results in graph theory, says that every planar graph can be represented as a coin graph, i.e., a contact graph of disks in the plane. In other words, given any planar graph with $n$ vertices, there is a set of $n$ disjoint open disks in the plane that are in one-to-one correspondence to the vertices such that a pair of disks is tangent if and only if the corresponding vertices are adjacent. Koebe's theorem has been rediscovered several times, see the survey of Sachs 12 . Collins and Stephenson 4 give an efficient algorithm for numerically approximating the radii and locations of the disks of such a

\footnotetext{
* Partially supported by projects PAI FQM-0164 and ORI MTM2005-08441-C02-01.

** Supported by grant WO 758/4-2 of the German Research Foundation (DFG). 


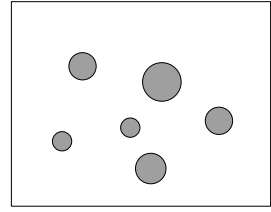

(a) disk seeds

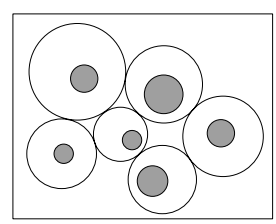

(b) disk cover of (a)

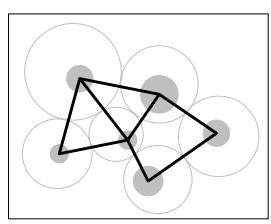

(c) CCG induced by (b)

Fig. 1. Seeds, cover, and CCG

representation of a planar graph. Their algorithm relies on an iterative process suggested by Thurston 13 .

Since Koebe there has been a lot of work in the graph-drawing community dedicated to the question which planar graphs can be represented as contact or intersections graphs of which geometric object. As a recent example, Fraysseix and Ossona de Mendez [5] showed that any four-colored planar graph without an induced four-colored $C_{4}$ is the intersection graph of a family of line segments.

On the other hand, there has been a lot of work in the geometric-optimization community dedicated to the question how to (optimally) cover geometric objects (usually points) by other geometric objects (like convex shapes, disks, annuli). As an example take Welzl's famous randomized algorithm [15] for finding the smallest enclosing ball of a set of points.

In this paper we combine the two previous problems: we are looking for geometric objects (like disks or triangles) whose interiors are disjoint, that cover given pairwise disjoint objects called seeds (like points or disks) and at the same time represent a given graph or graph property by the way they touch each other. Other than in geometric optimization each of our covering objects contains only one of the seeds. We are not interested in maximizing the sizes of the covering objects; instead we want them to jointly fulfill some graph-theoretic property (like connectivity). Compared to previous work on geometric representation of graphs we are more restricted in the choice of our representatives.

Let us get a bit more formal. Given a set $S$ of pairwise disjoint seeds of some type, a cover of $S$ is a set $\mathcal{C}$ of closed objects of some type with the property that each object contains exactly one seed and that the interiors of no two objects intersect. Figure 1b depicts a disk cover of the disk seeds in Figure 1a Now the cover contact graph (CCG) induced by $\mathcal{C}$ is the contact graph of the elements of $\mathcal{C}$. In other words, two vertices of a CCG are adjacent if the corresponding cover elements touch, i.e., their boundaries intersect. Figure 1c depicts the CCG induced by the cover in Figure $1 \mathrm{~b}$. Note that the vertices of the CCG are in one-to-one correspondence to both seeds and cover elements. We consider seeds to be topologically open (except if they are single points). Then seeds can touch each other. (Note that we require cover objects to be closed. This makes sure that a cover actually contains a point seed that lies on its boundary.)

In this paper we investigate the following questions.

Connectivity: Given a seed set, does it have a (1- or 2-) connected CCG? 
Realizability: Given a planar graph and a set of seeds, can the given graph be realized as a CCG on the given seeds?

A third type of question is treated in the long version of this article [3]:

Enumeration: For a given number of vertices, how many graphs of a certain graph class can be realized as a CCG?

However, we do consider in this paper an interesting restriction of the above problems where seeds and cover elements must lie in the half plane $\mathbb{R}_{+}^{2}$ above and including the $x$-axis. Seeds are additionally restricted in that each must contain at least one point of the $x$-axis. In this restricted setting we call the contact graph of a cover a $\mathrm{CCG}^{+}$. See Figures $7 \mathrm{~b}$ and 9 for examples.

Our results. First, we consider arbitrary sets of point seeds, see Section 2 Concerning connectivity we show that we can always cover a set of point seeds using disks or using homothetic triangles such that the resulting CCG is 1- or even 2-connected. Our algorithms run in $O(n \log n)$ expected and $O\left(n^{2}\right)$ worst-case time, respectively. Concerning realizability we give some necessary conditions and then show that it is NP-hard to decide whether a given graph can be realized as a disk-CCG if the correspondence between vertices and point seeds is given. Second, we consider the restriction where we are given a set $S$ of points on the $x$-axis as seeds. We show that in this case 1 -connectivity is easy: we can realize $C_{n}$ as a CCG on $S$ and there are trees that can be realized as a $\mathrm{CCG}^{+}$on $S$. For the case that the correspondence between seeds and vertices is given, we give an algorithm that decides in $O(n \log n)$ time which trees can be realized as $\mathrm{CCG}^{+}$. Third, we consider disk seeds, see Section 4 . We show that even deciding whether a set of disk seeds has a connected disk-CCG is NP-hard. We can only sketch proofs here. We refer the reader to the long version [3] of this paper.

Related work. Abellanas et al. 1] proved that the following problem, which they call the coin placement problem, is NP-complete. Given $n$ disks of varying radii and $n$ points in the plane, is there a way to place the disks such that each disk is centered at one of the given points and no two disks overlap?

Abellanas et al. 2] considered a related problem. They showed that given a set of points in the plane, it is NP-complete to decide whether there are disjoint disks centered at the points such that the contact graph of the disks is connected.

Given a pair of touching (convex) cover elements, we can draw the corresponding edge in the CCG by a two-segment polygonal line that connects the incident seeds and uses the contact point of the cover elements as bend. This is a link to the problem of point-set embeddability. We say that a planar graph $G$ is $k$-bend (point-set) embeddable if for any point set $P \subset \mathbb{R}^{2}$ there is a one-toone correspondence between $V$ and $P$ such that the edges of $G$ can be drawn as non-crossing polygonal lines with at most $k$ bends. Kaufmann and Wiese [8] showed that (a) every 4-connected planar graph is 1-bend embeddable, (b) every planar graph is 2-bend embeddable, and (c) given a planar graph $G=(V, E)$ and a set $P$ of $n$ points on a line, it is NP-complete to decide whether $G$ has a 1-bend embedding that maps $V$ one-to-one on $P$. 


\section{The Seeds Are Points in the Plane}

In this section we study point seeds which may take any position in the plane. If not stated otherwise our results hold for both disk covers and (homothetic) triangle covers. We focus on the two questions raised before: connectivity and realizability.

\subsection{Connectivity}

It is known to be NP-hard to decide whether a given set of points can be covered by a set of pairwise disjoint open disks, each centered on a point, such that the contact graph of the disks is connected [2]. In contrast to that result we give a simple sweep-line algorithm that covers point seeds by (non-centered) disks such that their contact graph is connected.

Proposition 1. Every set $S$ of $n$ point seeds has a connected $C C G$. Such a $C C G$ can be constructed in $O(n \log n)$ time and linear space.

Proof. After sorting $S$ by decreasing ordinate we proceed incrementally from top to bottom. For the first point, we place a cover element (disk or triangle, depending on the case) of fixed size with the seed as its bottommost point. If the $k-1$ topmost points are already connected, then for the $k$-th point $p$ we inflate a cover element $C_{p}$ with $p$ as the bottommost point until $C_{p}$ touches one of the previously placed cover elements.

The implementation for disk-CCGs is similar to Fortune's sweep [6] for constructing the Voronoi diagram of a set of weighted points. For triangle-CCGs we repeatedly determine the size of the new triangle in $O(\log n)$ time by a segmentdragging query [10] and two very simple ray-shooting queries.

In fact, even more can be obtained as the following proposition assures.

Proposition 2. Any set $S$ of $n$ point seeds has a biconnected CCG. Such a $C C G$ can be constructed in $O\left(n^{2} \log n\right)$ time using linear space.

Proof. We first consider disks as cover elements. Let $D_{1}, D_{2}$, and $D_{3}$ be three congruent disks that touch each other. They delimit a pseudo-triangular shape $R$. Choose the three disks such that each disk $D_{i}$ contains a unique point $p_{i} \in S$ and such that $S \backslash\left\{p_{1}, p_{2}, p_{3}\right\} \subset R$, see Figure 2 (left).

In order to cover the remaining points we assume that disks $D_{4}, \ldots, D_{i-1}$ have been placed such that each covers a unique point of $S$ and touches two previously placed disks, see Figure 2 (middle). Thus the contact graph of $D_{1}, \ldots, D_{i-1}$ is biconnected. Let $R_{j}$ be a connected component of $R \backslash \bigcup_{j=4}^{i-1} D_{i}$ that contains at least one uncovered point. Use Fortune's sweep [6] to compute the combined Voronoi diagram of the disks incident to $R_{j}$ and the points in $S \cap R_{j}$. This takes $O(n \log n)$ time and the resulting Voronoi diagram has complexity $O(n)$. The part of the Voronoi diagram in $R_{j}$ is the locus of the centers of all disks that lie in $R_{j}$ and touch $\partial R_{j} \cup\left(S \cap R_{j}\right)$ in at least two points, where $\partial R_{j}$ is the boundary of $R_{j}$. Now we make a simple but crucial observation: if $D$ is a disk that (a) lies 

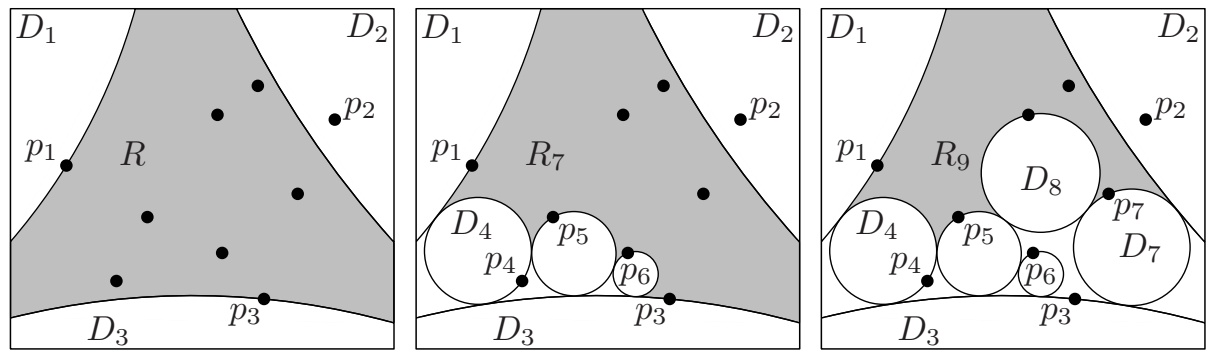

Fig. 2. Three steps in the construction of a biconnected disk-CCG

in $R_{j}$, (b) contains a seed $s \in S \cap R_{j}$ on its boundary, and (c) touches two of the previous disks, then $D$ is centered at a vertex of the Voronoi diagram. Thus a disk $D^{\star}$ fulfilling (a)-(c) can be found in linear time and, by construction, does not contain any point of $S$ in its interior. (If by any chance all such disks touch more than one point of $S$, we re-start the whole computation with three slightly wiggled initial disks $D_{1}, D_{2}$, and $D_{3}$. Then the probability of this degeneracy becomes 0 .) Now set $D_{i}=D^{\star}$, and repeat the process until all seeds are covered. This takes $O\left(n^{2} \log n\right)$ time in total.

The case of triangles can be handled analogously. Choosing any reference point in the triangular shape, a structure similar to the medial axis can be computed in $O(n \log n)$ and updated in $O(n)$ time in each of the $n-3$ phases.

\subsection{Realizability}

In this section we first give two necessary conditions that a planar graph must fulfill in order to be realizable as a disk-CCG on a given seed set. Then we construct a plane geometric graphs on six vertices that cannot be represented as disk-CCG. Finally we investigate the complexity of deciding realizability.

To formulate our necessary conditions for realizability we define a graph on the given seed set $S$. Our graph is inspired by the sphere-of-influence graph defined by Toussaint [14. Given a seed set $S$ and a point $p \in S$ let the influence area of $p$ be the closure of the union of all empty open disks $D$ (i.e., $D \cap S=\emptyset$ ) that are centered at vertices of the Voronoi region of $p$, see Figure 3 . We call the intersection graph of these influence areas the hyperinfluence graph of $S$ and denote it by $H I(S)$, see Figure 4

Proposition 3. Let $S$ be a set of point seeds and let $G$ be a graph realizable as a disk-CCG on $S$. Then

(i) $G$ is a subgraph of $H I(S)$, and

(ii) $G$ has a plane drawing where each vertex is mapped to a unique point in $S$ and each edge is drawn as a polygonal line with at most two segments (i.e., with at most one bend per edge). 


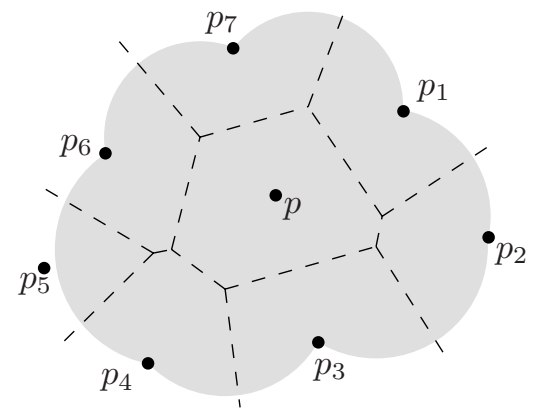

Fig. 3. Influence area of $p \in S$ (shaded)

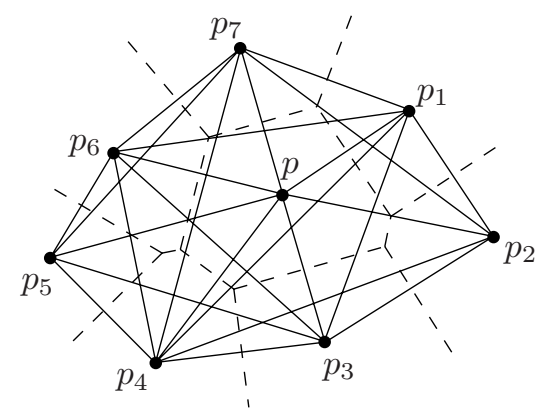

Fig. 4. The hyperinfluence graph $H I(S)$

Proof. Both facts are straightforward to obtain. (ii) is based on the observation that any possible covering disk of $p$ is contained in the influence area of $p$. Thus, if the covering disks of two seeds are in contact, their influence areas intersect.

(iii) is obtained by representing each edge of the CCG by two line segments that connect the seeds with the point of tangency of the covering disks.

While Proposition 3 (iii) is difficult to verify even if all seeds lie on a line 8 , Proposition 3 (1) gives us a way to show non-realizability of certain geometric graphs as the one depicted in Figure 5. That graph is connected and thus cannot be realized as a CCG with its vertices as seeds, because the shaded influence areas of $p_{1}$ and $p_{2}$ do not intersect. The graph has eight vertices. On the other hand it is easy to see that any three-vertex graph can be realized on any three-point seed set. Now it is interesting to ask for the least $n$ for which there is an $n$-vertex geometric graph $G$ such that the straight-line drawing of $G$ is plane but $G$ cannot be realized as CCG.

We show that there is a set $S=\{a, b, \ldots, f\}$ of six points in convex position such that their Delaunay triangulation is not representable as a CCG, see the underlying graph in Figure 6. The covering disks $D_{a}$ and $D_{d}$ of the points $a$ and $d$ must touch each other in one of two ways. Either the tangent point of the disks lies inside the convex hull of $S$, or $D_{a}$ and $D_{d}$ are very large and lie to the left

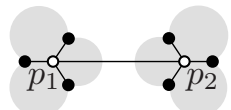

Fig. 5. Non-realizable bipartite graph of $a$ and to the right of $d$, in which case they touch far above or below $S$, see Figure [6. In the first case there is no disk covering $c$ and touching $D_{a}$. In the second case we can assume that the boundaries of $D_{a}$ and $D_{d}$ are two almost parallel lines in the vicinity of the six points. The disks $D_{c}$ and $D_{f}$ covering $c$ and $f$ must both touch $D_{a}$ and $D_{d}$. But if $c$ and $f$ are close enough to $a$ and $d$ then $D_{c}$ and $D_{f}$ cannot be disjoint.

So we have seen that there are pairs of (quite small) graphs and seed sets such that the graph cannot be realized on the seed set as disk CCG. Thus we would like to decide whether a given graph is realizable as CCG on a given seed set or not. Of course Koebe's theorem 9] guarantees that for any planar graph $G$ we can find a seed set $S$ such that it is possible to realize $G$ on $S$. However, if 

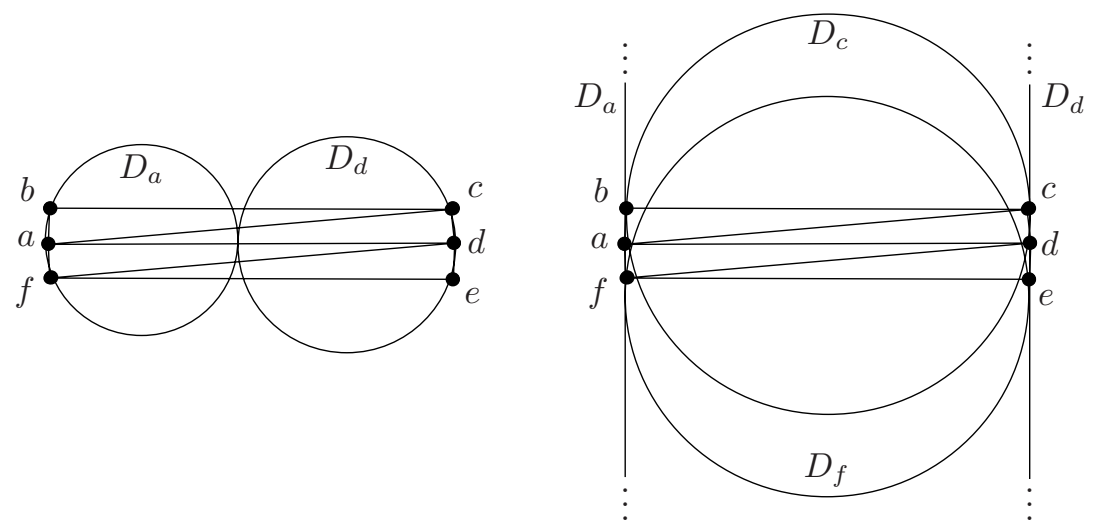

Fig. 6. Non-realizable Delaunay triangulation of six points in convex position

the seeds and the vertex-seed correspondence are given, the problem becomes NP-hard.

Theorem 1. Given a set $S$ of points in the plane and a planar graph $G=(S, E)$, it is NP-hard to decide whether $G$ is realizable as disk-CCG on $S$.

The proof is by reduction from the NP-hard problem PLANAR3SAT. There are gadgets for each variable and each clause of the given Boolean formula. The gadget of a variable $v$ is such that it allows two combinatorially different ways to represent the given subgraph as disk-CCG. These correspond to the two Boolean values of $v$. The clause gadget is locally symmetric with respect to $120^{\circ}$-rotations and designed such that some cover disks must overlap if and only if the corresponding three literals are all false.

\section{The Seeds Are Points on a Line}

In this section, seed sets consist of points on the $x$-axis. Connectivity follows from some of our realizability results, so we focus on the latter. We consider the following four questions. Note that seeds now correspond to real numbers, so we can use the natural order $<$ in $\mathbb{R}$ to compare them. All covers consist of disks unless stated otherwise (e.g., in Q44).

Q1. Given a graph class $\mathcal{C}$ (e.g., the class of trees), does it hold that for any seed set $S$ there is a graph in $\mathcal{C}$ that is realizable as $\mathrm{CCG}$ or $\mathrm{CCG}^{+}$on $S$ ?

We show: This is true for (cycles, CCG) and (trees, $\mathrm{CCG}^{+}$).

Q2. Given a graph class $\mathcal{C}$, does it hold that for any graph $G$ in $\mathcal{C}$ there is a seed set $S$ such that $G$ can be realized as CCG or $\mathrm{CCG}^{+}$on $S$ ?

We show: This is true for the combination (trees, $\mathrm{CCG}^{+}$).

Q3. Let $\mathcal{C}$ be a fixed graph class. Given a graph $G \in \mathcal{C}$ with a labeling $\lambda: V \rightarrow$ $\{1, \ldots, n\}$, is there a sequence $s_{1}<\ldots<s_{n}$ of seeds in $\mathbb{R}^{1}$ and a realization of $G$ that maps each vertex $v$ to the corresponding seed $s_{\lambda(v)}$ ?

We show: There is an $O(n \log n)$ decision algorithm for (trees, $\left.\mathrm{CCG}^{+}\right)$. 


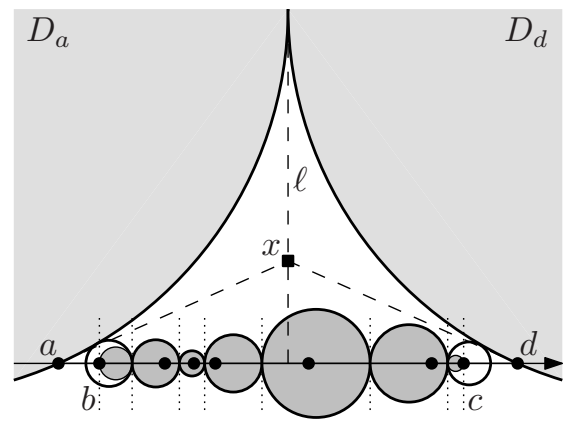

(a) $C_{n}$ is realizable as $\mathrm{CCG}$

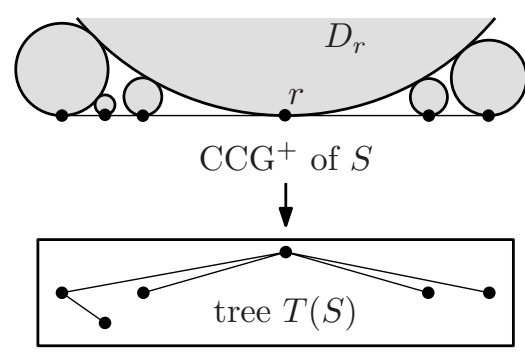

(b) tree $T(S)$ is realizable as $\mathrm{CCG}^{+}$

Fig. 7. Graphs that can be realized on a given one-dimensional $n$-point seed set $S$

Q4. Let $\mathcal{C}$ be a fixed graph class. Given a seed set $S$ and a graph $G(S, E) \in \mathcal{C}$, can $G$ be realized on $S$ as triangle $\mathrm{CCG}$ or $\mathrm{CCG}^{+}$?

We show: There is an $O(n \log n)$-time decision algorithm for (trees, $\left.\mathrm{CCG}^{+}\right)$.

Note that the above questions require more and more concrete information about the seed set, ranging from no information $(\mathrm{Q} 2$ ) via a fixed order $(\mathrm{Q} 3 \mathrm{3})$ to complete information (Q4). We start with question Q1]

Proposition 4. Let $S$ be a set of $n$ point seeds on a line, then

(i) the $n$-vertex cycle $C_{n}$ can be realized as $C C G$ on $S$, and

(ii) there is a tree $T(S)$ that can be realized as $C C G^{+}$on $S$.

Figures $7 \mathrm{a}$ and $7 \mathrm{~b}$ give some intuition about how our algorithms work; for details see the long version of this paper [3].

In terms of this paper, a coin graph is obtained when seeds are points and cover elements are disks centered at seeds, and thus Koebe's theorem establishes that it is always possible to choose seeds in the plane such that any given plane graph is realizable as a coin graph on them. We have seen in Proposition 4 that $C_{n}$ is realizable as a CCG on any seed set on a line. One can ask whether a Koebe-type theorem also holds in this restricted setting. However, Kaufmann and Wiese [7 have shown that there is a plane triangulated 12-vertex graph (see Figure 8) that cannot be drawn with only one bend per edge if vertices are restricted to a line. Now Proposition 3 (iii) implies that that graph is not realizable as CCG if seeds lie on a line. On the positive side, we can show that a Koebe-type theorem holds for the combination (trees, $\mathrm{CCG}^{+}$). This is an answer to Q2 and in a way dual to Proposition 4 (iii). See Figure 9 for a sketch of our recursive construction.

Proposition 5. For any tree $T$ there is a seed set $S(T) \subset \mathbb{R}^{1}$ such that $T$ is realizable as $C C G^{+}$on $S(T)$. 


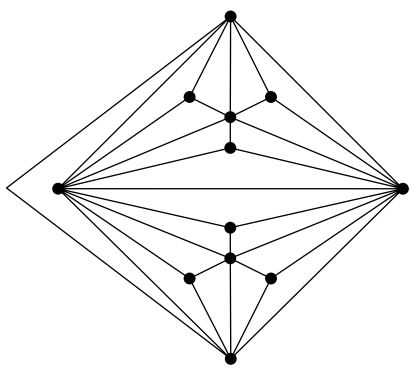

Fig. 8. Kaufmann-Wiese graph 8

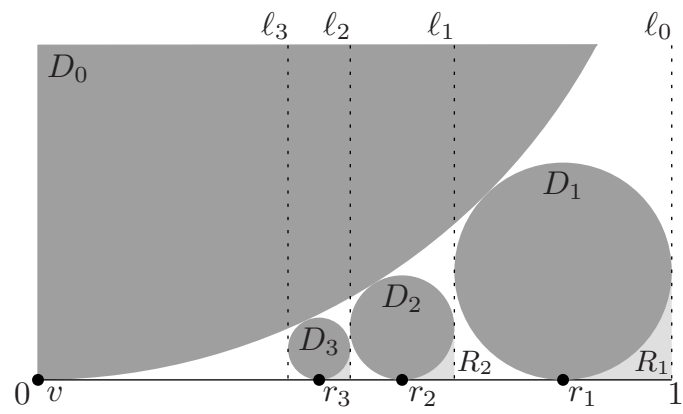

Fig. 9. Constructing a seed set $S(T)$

In Proposition 5 above, we had complete freedom to choose the seeds. Now we turn to question Q 3 , where we are not just given a tree, but also an order of its vertices that must be respected by the corresponding seeds. Kaufmann and Wiese 7] have investigated a related problem. They showed that it is NP-complete to decide whether the vertices of a given (planar) graph can be put into one-to-one correspondence with a given set of points on a line such that there is a plane drawing of the graph with at most one bend per edge. We call such a drawing a 1d-1BD. If additionally all bends lie on one side of the line, we call the drawing a $1 \mathrm{~d}-1 \mathrm{BD}^{+}$.

Note that the hardness result of Kaufmann and Wiese does not yield the hardness of the one-dimensional CCG realizability problem, since not every graph that can be one-bend embedded on a set of points on a line is realizable as $\mathrm{CCG}$, let alone as $\mathrm{CCG}^{+}$. Our next result explores the gap between Kaufmann and Wiese's one-dimensional embeddability problem and the situation in Proposition 5 .

More formally, given an $n$-vertex tree $T$ and a (bijective) labeling $\lambda: V \rightarrow$ $\{1, \ldots, n\}$ of its vertices, we say that $T$ is $\lambda$-realizable (as $\mathrm{CCG}, \mathrm{CCG}^{+}, 1 \mathrm{~d}$ $\left.1 \mathrm{BD}, 1 \mathrm{~d}-1 \mathrm{BD}^{+}\right)$if there is a sequence $s_{1}<\ldots<s_{n}$ of seeds in $\mathbb{R}^{1}$ and a realization of $T$ (as $\mathrm{CCG}, \mathrm{CCG}^{+}, 1 \mathrm{~d}-1 \mathrm{BD}, 1 \mathrm{~d}-1 \mathrm{BD}^{+}$) that maps each vertex $v$ to the corresponding seed $s_{\lambda(v)}$.

In order to obtain a characterization of trees that are $\lambda$-realizable as $\mathrm{CCG}^{+}$, we need the following definition. Given a graph $G=(V, E)$ with vertex labeling $\lambda$, a forbidden pair is a pair of edges $\{\{a, b\},\{c, d\}\}$ such that $\lambda(a)<\lambda(c)<$ $\lambda(b)<\lambda(d)$. Note that it is impossible to embed the edges of a forbidden pair simultaneously above the $x$-axis.

Theorem 2. For a $\lambda$-labeled tree $T$ the following statements are equivalent:

(i) $T$ is $\lambda$-realizable as a $C C G^{+}$.

(ii) $T$ is $\lambda$-realizable as a $1 d-1 B D^{+}$.

(iii) $T$ does not contain any forbidden pair.

Given the tree, statement (iii) can be checked in $O(n \log n)$ time using an interval tree, therefore the following corollary is straightforward. 

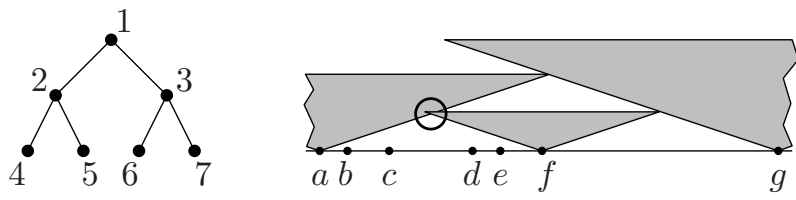

Fig. 10. Binary tree not realizable as $\mathrm{CCG}^{+}$on given seeds

Corollary 1. Given a $\lambda$-labeled tree $T$, we can decide in $O(n \log n)$ time whether $T$ is $\lambda$-realizable as $C C G^{+}$.

We now turn to question Q44. So given a set of seeds $S$ and a tree $T(S, E)$ our answer is a decision algorithm for the realizability of $T$ as a triangle $\mathrm{CCG}^{+}$on $S$. Note that in our series of results about realizability we have required more and more concrete information about the seed set, ranging from no information (Proposition 5) via a fixed order (Theorem 2) to complete information now. We call a triangle $V$-shaped if it is symmetric to a vertical line and if its bottommost vertex is unique. In the following we will consider all triangles as $\mathrm{V}$-shaped. First note that there are trees $T$ and seed sets $S$ for which the answer to question Q4 4 is negative even if the mapping between vertices and seeds is not fixed in advance. Figure 10 shows a complete binary tree $T$ on seven vertices and the onedimensional point set $S=\{a(0), b(2), c(5), d(11), e(13), f(16), g(33)\}$. A case distinction on the seed that represents the root vertex 1 shows that it is not possible to find a representation of $T$ as a triangle $\mathrm{CCG}^{+}$on $S$. The example in Figure 10 shows the case where seed $g$ represents the root. In this case any two covers of points in $S \backslash\{g\}$ that touch the cover of $g$ will overlap, e.g., the covers of $a$ and $f$.

On the other hand, there is always a tree that can be realized on a given set of seeds as Proposition 4 (iii) shows. We can give an algorithm that decides this realizability for a pair $(S, T)$ with $T=(S, E)$ in $O(n \log n)$ time, where $n=|S|$.

Theorem 3. Given a set of seeds $S$ and a tree $T=(S, E)$ we can decide in $O(n \log n)$ time whether $T$ can be realized as a $V$-shaped triangle $C C G^{+}$on $S$.

The decision algorithm is based on the observation that the covers for the closest pair of seeds must touch each other as otherwise this $\mathrm{CCG}^{+}$would not be connected. Thus the algorithm adds the edge between the closest pair of seeds, removes one of the two seeds, and continues this process as long as it complies with $T$. We can use the same algorithm to generate all trees that can be realized as $\mathrm{CCG}^{+}$on $S$ by branching on the seed to remove in each iteration.

Although Theorem 3 is stated for a very restricted class of triangles, the result can easily be extended to homothetic triangles whose top sides are parallel to the $x$-axis.

\section{The Seeds Are Disks in the Plane}

In this section, we consider disks in the plane as seeds and cover them using disks, too. In contrast to point seeds the minimal size of each cover element is 


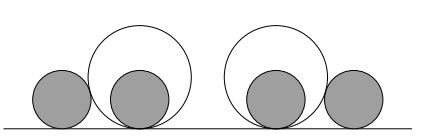

(a) seed set without connected $\mathrm{CCG}^{+}$

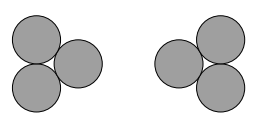

(b) seed set without connected CCG

Fig. 11. Disk seed sets without connected disk covers

now bounded from below by the size of the corresponding seed. Therefore the results in this section differ a lot from those obtained in previous sections.

Unlike the connectivity results for points we can neither guarantee the existence of a connected $\mathrm{CCG}^{+}$for disk seeds touching a line nor the existence of a connected CCG for disk seeds in the plane, see Figure 11 Deciding whether a given set of disk seeds has a connected CCG turns out to be hard.

Theorem 4. Given a set $S$ of disk seeds, it is NP-hard to decide whether there is a connected $C C G$ on $S$, even if there are only four different seed radii.

The proof is again by reduction from Planar3SAT. The main trick is to use what we call a stopper element, a cluster of three congruent pairwise touching disks as in Figure 11b. Observe that these disks can only be covered by themselves - any larger cover of any disk would intersect the others. We use small copies of these stopper elements to discretize the way in which other seeds can be covered. In the center of our clause gadget there is stopper element that is connected to the remaining cover as long as any of the corresponding three literals is true.

Concerning realizability, the hardness result of Theorem 1 clearly still holds for disk seeds. The necessary conditions for realizability in Proposition 3 can be adapted to the case of disk seeds.

\section{Open Problems}

This paper has opened a new field with many interesting questions.

1. We know that every 3-vertex graph can be represented as CCG on any set of three points. We have given an example of six points whose Delaunay triangulation is not representable as a CCG. What about plane geometric graphs with four or five vertices? Do they always have a representation?

2. Does any set of point seeds in convex position have a triangulation that can be represented as CCG?

3. We know that any set of point seeds has a 2-connected CCG. What about 3 -connectivity?

4. Is it NP-hard to decide whether a set of disks touching a line has a connected $\mathrm{CCG}^{+}$?

5. Is there an equivalent to Theorem 2 for CCG's, i.e., can we characterize vertex-labeled trees that have a realization as CCG on a set of seeds on a line which respect the vertex order prescribed by the labeling?

6. What about other classes of seeds and covers? 


\section{References}

1. Abellanas, M., Bereg, S., Hurtado, F., Olaverri, A.G., Rappaport, D., Tejel, J.: Moving coins. Comput. Geom. Theory Appl. 34(1), 35-48 (2006)

2. Abellanas, M., de Castro, N., Hernández, G., Márquez, A., Moreno-Jiménez, C.: Gear system graphs. Manuscript (2006)

3. Atienza, N., de Castro, N., Cortés, C., Garrido, M. Á., Grima, C.I., Hernández, G., Márquez, A., Moreno, A., Nöllenburg, M., Portillo, J.R., Reyes, P., Valenzuela, J., Villar, M.T., Wolff, A.: Cover contact graphs. Technical Report 2007-18, Fakultät für Informatik, Universität Karlsruhe (September 2007), http://www.ubka.uni-karlsruhe.de/indexer-vvv/ira/2007/18

4. Collins, C.R., Stephenson, K.: A circle packing algorithm. Comput. Geom. Theory Appl. 25(3), 233-256 (2003)

5. de Fraysseix, H., de Mendez, P.O.: Representations by contact and intersection of segments. Algorithmica 47(4), 453-463 (2007)

6. Fortune, S.: A sweepline algorithm for Voronoi diagrams. In: SoCG 1986. Proc. 2nd Annu. Sympos. Comput. Geom., pp. 313-322 (1986)

7. Giménez, O., Noy, M.: The number of planar graphs and properties of random planar graphs. In: Martínez, C. (ed.) ICAA 2005. Proc. Internat. Conf. Anal. Algorithms, DMTCS Proceedings, vol. AD, pp. 147-156 (2005)

8. Kaufmann, M., Wiese, R.: Embedding vertices at points: Few bends suffice for planar graphs. J. Graph Algorithms Appl. 6(1), 115-129 (2002)

9. Koebe, P.: Kontaktprobleme der konformen Abbildung. Ber. Sächs. Akad. Wiss. Leipzig, Math.-Phys. Klasse 88, 141-164 (1936)

10. Mitchell, J.S.B.: $\mathrm{L}_{1}$ shortest paths among polygonal obstacles in the plane. Algorithmica 8, 55-88 (1992)

11. Pach, J., Agarwal, P.K.: Combinatorial Geometry. John Wiley and Sons, New York (1995) (contains a proof of Koebe's theorem)

12. Sachs, H.: Coin graphs, polyhedra, and conformal mapping. Discrete Math. 134(13), 133-138 (1994)

13. Thurston, W.P.: The Geometry and Topology of 3-Manifolds. Princeton University Notes, Princeton (1980)

14. Toussaint, G.T.: A graph-theoretical primal sketch. In: Toussaint, G.T. (ed.) Computational Morphology: A Computational Geometric Approach to the Analysis of Form, North-Holland, pp. 229-260 (1988)

15. Welzl, E.: Smallest enclosing disks (balls and ellipsoids). In: Maurer, H.A. (ed.) New Results and New Trends in Computer Science. LNCS, vol. 555, pp. 359-370. Springer, Heidelberg (1991) 